\title{
QUICKLY UNKNOTTING TOPOLOGICAL SPHERES
}

\author{
W. R. BRAKES
}

\begin{abstract}
Locally flat topological sphere and cell pairs of codimension at least three are shown to be unknotted by a simpler method than previously known.
\end{abstract}

Let $f: S^{r} \rightarrow S^{n}$ be a locally flat embedding, where $n-r \geqslant 3$. This paper demonstrates the existence of a homeomorphism $h$ of $S^{n}$ such that $h\left(S^{r}\right)=$ $f\left(S^{r}\right)$, where $S^{r}$ is viewed as a submanifold of the $n$-sphere $S^{n}$ by considering $S^{n}$ as the join $S^{r} * S^{n-r-1}$. It then follows easily that $h^{-1} f$ extends to a homeomorphism of $S^{n}$, by setting the extension equal to the identity on $S^{n-r-1}$ and extending joinwise, and so $f$ extends to a homeomorphism of $S^{n}$.

The method of proof simplifies the known procedure due to Stallings [8] in that it requires just one straightforward application of his engulfing lemma, thus greatly reducing the algebraic technicalities. Although something is lost in this simplification, namely Stallings' results on codimension two embeddings, there is also a gain: the dimension restriction $(n \geqslant 5)$ of Stallings disappears, and so the unknotting of $S^{1}$ in $S^{4}[6]$ is included in a general theory without recourse to any piecewise linear approximation. A similar unknotting theorem for cell pairs is also obtained.

$B^{n}$ will denote the unit ball in $R^{n}$, euclidean $n$-space, and for $r<n$ a fixed standard inclusion $R^{r} \subset R^{n}$ is assumed which restricts to a similar inclusion $B^{r} \subset B^{n}$. $\mathrm{Cl}$ and Int will denote closure and interior respectively, and $X \subset \subset Y$ will mean $\mathrm{Cl} X \subset$ Int $Y$.

The crux of the proof is the following lemma, a pairwise Schoenflies theorem which should have further applications. The statement of this lemma is best grasped by viewing $S^{n}, S^{n-1}$ and $S^{r}$ in the following slightly nonstandard way: $S^{n-1}$ is the equator of $S^{n}$ and $S^{r}$ is an $r$-dimensional subsphere of $S^{n}$ passing through the poles and so is not a subset of $S^{n-1}$.

Lemma (Pairwise Schoenflies Theorem). If $f: S^{n-1} \times I \rightarrow S^{n}$ is an embedding, with

$$
f\left(S^{n-1} \times I\right) \cap S^{r}=f\left(S^{r-1} \times I\right)
$$

$[I \equiv[0,1]]$, then the closures of the complementary domains of $f\left(S^{n-1} \times 1 / 2\right)$ are $n$-balls, their intersections with $S^{r}$ being standard $r$-dimensional subballs.

Proof. The generalised Schoenflies theorem implies that the closures of the complementary domains of $f\left(S^{n-1} \times 1 / 2\right)$ are $n$-balls, and likewise that their

Received by the editors September 19, 1975 and, in revised form, September 12, 1977. AMS (MOS) subject classifications (1970). Primary 57A35; Secondary 57A30. 
intersections with $S^{r}$ are $r$-dimensional subballs. The strength of the statement of the lemma is that they are standard (i.e. unknotted) subballs. This is proved by following through M. Brown's proof of the Schoenflies theorem, while keeping a tight hold throughout on intersections with $S^{r}$. Thus the crucial step is to establish that if $A$ is the closure of one component of $S^{n}-f\left(S^{n-1} \times I\right)$ then $A$ is cellular in $S^{n}$, with, for each $n$-ball $Y_{m}$ in the sequence, $\left(Y_{m}, Y_{m} \cap S^{r}\right)$ pairwise homeomorphic to $\left(B^{n}, B^{r}\right)$. The proof will then be completed via Brown's shrinking device, again applied with care.

Let $Y$ be the closure of the complement in $S^{n}$ of a 'small, round' ball neighbourhood of $f\left(x_{0}, \frac{1}{2}\right)$, for some point $x_{0}$ in $S^{r-1}$, ensuring that $Y$ is an $n$-ball with $S^{r} \cap Y$ a standard $r$-dimensional subball. Let $A$ and $B$ be the closures of the two components of $S^{n}-f\left(S^{n-1} \times I\right)$, with $f\left(S^{n-1} \times 0\right)$ as the frontier of $A$, and write $S^{n}$ as $\{a, b\} * S^{n-1}$, with $a$ and $b$ chosen in $S^{r}$ so that $S^{r}=\{a, b\} * S^{r-1}$. Define $g: S^{n} \rightarrow S^{n}$ by $g(A)=a, g(B)=b$,

$$
\begin{array}{ll}
g f(x, t)=(1-2 t) a+2 t x & \text { if } 0 \leqslant t \leqslant 1 / 2, \\
g f(x, t)=(2 t-1) b+(2-2 t) x & \text { if } 1 / 2 \leqslant t \leqslant 1 .
\end{array}
$$

Note that $g\left(S^{r}\right)=S^{r}$. Let $F$ be a homeomorphism of $S^{n}$ such that $F g($ Int $Y)$ contains $b$ but $F g(Y)$ does not contain $a, F\left(S^{r}\right)=S^{r}$, and $F$ is the identity on a neighbourhood of $b$. Define $G: Y \rightarrow S^{n}$ by $G(x)=x$ if $x \in B, G(x)=$ $g^{-1} F g(x)$ if $x \notin B . G$ is continuous and one-one except that $G(A)=$ $g^{-1} F(a)=y_{0}$, say, a point in $S^{r} \cap f\left(S^{n-1} \times I\right)$. Now, let $U$ be an arbitrary neighbourhood of $A$, so $G(U)$ is a neighbourhood of $y_{0}$. Let $H$ be a homeomorphism of $S^{n}$ such that $H G(Y) \subset G(U), H\left(S^{r}\right)=S^{r}$ and $H$ is the identity on a neighbourhood of $y_{0}$. Define $h: Y \rightarrow S^{n}$ by $h(x)=x$ if $x \in A$, $h(x)=G^{-1} H G(x)$ if $x \notin A$. It is clear that $h$ is an embedding, and if $Z=h(Y)$, then $A \subset \subset Z \subset \subset U$ and $\left(Z, Z \cap S^{r}\right)$ is pairwise homeomorphic to $\left(B^{n}, B^{r}\right)$. So $A$ is cellular in $S^{n}$, with each ball concerned in the sequence having its intersection with $S^{r}$ a standard $r$-dimensional subball.

Let $X$ be the closure of that component of $S^{n}-f\left(S^{n-1} \times \frac{1}{2}\right)$ containing $A$. Then $X$ is homeomorphic to $X / A$ by the usual shrinking method (Theorem 1 of [2]). $S^{r}$ is preserved setwise under each such shrinking, so under this homeomorphism $X \cap S^{r} \rightarrow q\left(X \cap S^{r}\right)$ where $q: X \rightarrow X / A$ is the quotient map. But $X / A$ is homeomorphic to $B^{n}$ by a homeomorphism induced by $g \mid X$, and since $g$ also preserves $S^{r}$ setwise, $q\left(X \cap S^{r}\right) \rightarrow B^{r}$ under this homeomorphism. Thus $\left(X, X \cap S^{r}\right)$ is pairwise homeomorphic to $\left(B^{n}, B^{r}\right)$ as required.

THEOREM. If $f: S^{r} \rightarrow S^{n}$ is a locally flat embedding where $n-r \geqslant 3$, then there exists a homeomorphism $h$ of $S^{n}$ such that $h\left(S^{r}\right)=f\left(S^{r}\right)$.

Proof. Let $x_{0}$ be any point of $S^{r}$ and let $N$ be a neighborhood of $f\left(x_{0}\right)$ in $S^{n}$, guaranteed by local flatness, such that for some homeomorphism $g$ : $R^{n} \rightarrow N, g\left(R^{r}\right)=N \cap f\left(S^{r}\right)$. Let $V, W$ denote respectively $f^{-1} g\left(B^{r}\right)$, 
$f^{-1} g\left(2 B^{r}\right) . \mathrm{Cl}\left(S^{r}-V\right)$ is a locally flat $r$-ball in $S^{n}$; it is therefore flat by [7], so it may be assumed that $f \mid \mathrm{Cl}\left(S^{r}-V\right)=$ identity. Applying Stallings' engulfing lemma (Theorem 6.1 of [8]) with

$$
\begin{array}{ll}
M=S^{n}-\mathrm{Cl}\left(S^{r}-W\right), & U=N-\mathrm{Cl}\left(S^{r}-W\right), \\
C=\text { Int } W-\text { Int } V, & P=\text { Int } W,
\end{array}
$$

produces a homeomorphism of $M$ which extends by the identity on $\mathrm{Cl}\left(S^{r}-\right.$ $W)$ to a homeomorphism $e$ of $S^{n}$ satisfying $e \mid \mathrm{Cl}\left(S^{r}-V\right)=$ identity and $V \subset e(N)=e g\left(R^{n}\right)$. So if $K$ is large enough, $V \subset \subset \operatorname{eg}\left(K B^{n}\right)$. Then if $e g \mid \mathrm{Cl}\left((K+1) B^{n}-K B^{n}\right)$ is viewed as an embedding $S^{n-1} \times I \rightarrow S^{n}$, it satisfies

$$
e g\left(S^{n-1} \times I\right) \cap S^{r}=e g\left(S^{r-1} \times I\right) .
$$

So, by the Lemma,

$$
e g \mid \mathrm{Cl}\left((K+1) B^{n}-(K+(1 / 2)) B^{n}\right)
$$

extends to an embedding $G:(K+1) B^{n} \rightarrow S^{n}$ such that

$$
G\left((K+1) B^{n}\right) \cap S^{r}=G\left((K+1) B^{r}\right) .
$$

Now,

$$
G(e g)^{-1}: e g\left((K+1) B^{n}\right) \rightarrow e g\left((K+1) B^{n}\right)
$$

is the identity on the boundary, so extends by the identity to a homeomorphism $h$ of $S^{n}$. Clearly $h\left(e f\left(S^{r}\right)\right)=S^{r}$, and so $(h e)^{-1}$ is the required homeomorphism.

The following corollary, an unknotting theorem for topological cell-pairs, was first obtained by Glaser and Price [5]. Again the original version established conditions for the unknotting of codimension two pairs, but did not contain the 1 in 4 case included here.

COROLLARY. If $f: B^{r} \rightarrow B^{n}$ is a locally flat proper embedding (i.e. $f^{-1}\left(S^{n-1}\right)$ $\left.=S^{r-1}\right)$ and $n-r \geqslant 3$, then $\left(B^{n}, f\left(B^{r}\right)\right)$ is pairwise homeomorphic to $\left(B^{n}, B^{r}\right)$.

Proof. Apply the theorem above to unknot the boundary sphere pair, and so assume that $f \mid S^{r-1}$ is the inclusion. Extend $f$ via the identity to an embedding $f: S^{r} \rightarrow S^{n}$ (viewing $S^{n}$ as the one-point compactification of $\left.R^{n} \supset B^{n}\right)$. Apply the theorem again to extend $f$ to a homeomorphism $F$ of $S^{n}$. Then

$$
\begin{aligned}
F^{-1}\left(\mathrm{Cl}\left(2 B^{n}-B^{n}\right)\right) \cap S^{r} & =\mathrm{Cl}\left(2 B^{r}-B^{r}\right) \\
& =F^{-1}\left(\mathrm{Cl}\left(2 B^{r}-B^{r}\right)\right) .
\end{aligned}
$$

So the Lemma can be applied to extend $F^{-1} \mid \mathrm{Cl}\left(2 B^{n}-(3 / 2) B^{n}\right)$ to an embedding

$$
G:\left(2 B^{n}, 2 B^{r}\right) \rightarrow\left(S^{n}, S^{r}\right),
$$

where $G\left(2 B^{r}\right)=2 B^{r}$, and $G$ can be extended by $F^{-1} \mid S^{n}-2 B^{n}$ to a 
homeomorphism (still called $G$ ) of $S^{n}$. Then $F G$ is the identity outside $2 B^{n}$ and

$$
F G\left(2 B^{r}\right)=F\left(2 B^{r}\right)=f\left(2 B^{r}\right) .
$$

That is, $F G:\left(2 B^{n}, 2 B^{r}\right) \rightarrow\left(2 B^{n}, f\left(2 B^{r}\right)\right)$. The proof is completed by applying the usual collar argument (using [4] or [3]) to show that $\left(2 B^{n}, f\left(2 B^{r}\right)\right)$ is pairwise homeomorphic to $\left(B^{n}, f\left(B^{r}\right)\right)$.

\section{REFERENCES}

1. W. R. Brakes, $k$-stability of homeomorphisms of euclidean n-space, Proc. Cambridge Philos. Soc. 75 (1974), 175-183.

2. M. Brown, A proof of the generalised Schoenflies theorem, Bull. Amer. Math. Soc. 66 (1960), $74-76$.

3. M. Brown, Locally flat embeddings of topological manifolds, Ann. of Math. 75 (1962), 331-342.

4. R. Conelly, A new proof of M. Brown's collaring theorem, Proc. Amer. Math. Soc. 27 (1971), 180-182.

5. L. C. Glaser and T. M. Price, Unknotting locally flat cell pairs, Illinois J. Math. 10 (1966), $425-430$.

6. H. Gluck, Unknotting $S^{1}$ in $S^{4}$, Bull. Amer. Math. Soc. 69 (1963), 91.

7. R. C. Lacher, Locally flat strings and half-strings, Proc. Amer. Math. Soc. 18 (1967), 299-304.

8. J. R. Stallings, On topologically unknotted spheres, Ann. of Math. 78 (1963), 490-503.

Mathematics Institute, University of Warwick, Coventry, CV4 7AL, ENGland

Current address: Faculty of Mathematics, The Open University, Walton Hall, Milton Keynes, MK7 6AA, England 